\title{
REALIZING ROTATION VECTORS FOR TORUS HOMEOMORPHISMS
}

\author{
BY JOHN FRANKS
}

AbSTRACT. We consider the rotation set $\rho(F)$ for a lift $F$ of a homeomorphism $f: T^{2} \rightarrow T^{2}$, which is homotopic to the identity. Our main result is that if a vector $v$ lies in the interior of $\rho(F)$ and has both coordinates rational, then there is a periodic point $x \in T^{2}$ with the property that

$$
\frac{F^{q}\left(x_{0}\right)-x_{0}}{q}=v
$$

where $x_{0} \in R^{2}$ is any lift of $x$ and $q$ is the least period of $x$.

In this article we consider the rotation set $\rho(F)$ as defined in [MZ], for a lift $F$ of a homeomorphism $f: T^{2} \rightarrow T^{2}$, which is homotopic to the identity. Our main result is that if a vector $v$ lies in the interior of $\rho(F)$ and has both coordinates rational, then there is a periodic point $x \in T^{2}$ with the property that

$$
\frac{F^{q}\left(x_{0}\right)-x_{0}}{q}=v
$$

where $x_{0} \in R^{2}$ is any lift of $x$ and $q$ is the least period of $x$. This should be compared with the well-known fact that if a homeomorphism of the circle has rational rotation number $p / q$ then it has a periodic point (with rotation number $p / q)$.

R. MacKay and J. Llibre [ML] have proved a similar result using the ideas our Proposition (2.4) below. They require the stronger hypothesis that $v$ is in the interior of the convex hull of vectors in $\rho(F)$ which represent periodic orbits of $f$.

\section{BACKGROUND AND DEFINITIONS}

Suppose $f: T^{2} \rightarrow T^{2}$ is a homeomorphism homotopic to the identity map, and let $F: R^{2} \rightarrow R^{2}$ be a lift.

(1.1) Definition. Let $\rho(F)$ denote the set of accumulation points of the subset of $R^{2}$

$$
\left\{\frac{F^{n}(x)-x}{n} \mid x \in R^{2}, n \in Z^{+}\right\},
$$

Received by the editors August 23, 1988.

1980 Mathematics Subject Classification (1985 Revision). Primary 58F22. 
thus $\nu \in \rho(F)$ if there are sequences $x_{i} \in R^{2}$ and $n_{i} \in Z^{+}$with $\lim n_{i}=\infty$ such that

$$
\lim _{i \rightarrow \infty} \frac{F^{n_{i}}\left(x_{i}\right)-x_{i}}{n_{i}}=\nu
$$

In [MZ] the rotation set is defined for a map homotopic to the identity (rather than a homeomorphism) $f: T^{n} \rightarrow T^{n}$. However, we shall be concerned only with homeomorphisms of $T^{2}$. In [MZ] it is shown that for homeomorphisms of $T^{2}, \rho(F)$ is convex.

We now briefly review the elementary theory of attractor-repeller pairs and chain recurrence developed by Charles Conley in [C]. In the following $f: X \rightarrow$ $X$ will denote a homeomorphism of a compact metric space $X$.

(1.2) Definition. An $\varepsilon$-chain for $f$ is a sequence $x_{1}, x_{2}, \ldots, x_{n}$ of points in $X$ such that

$$
d\left(f\left(x_{i}\right), x_{i+1}\right)<\varepsilon \quad \text { for } 1 \leq i \leq n-1 .
$$

If $x_{1}=x_{n}$ it is called a periodic $\varepsilon$-chain.

A point $x \in X$ is called chain recurrent if for every $\varepsilon>0$ there is an $n$ (depending on $\varepsilon$ ) and an $\varepsilon$-chain $x_{1}, x_{2}, \ldots, x_{n}$ with $x_{1}=x_{n}=x$. The set $\mathbf{R}$ of chain recurrent points is called the chain recurrent set of $f$.

It is easily seen that $\mathbf{R}$ is compact and invariant under $f$.

If $A \subset X$ is a compact subset and there is an open neighborhood $U$ of $A$ such that $f(\operatorname{cl}(U)) \subset U$ and $\bigcap_{n \geq 0} f^{n}(\operatorname{cl}(U))=A$, then $A$ is called an attractor and $U$ is its isolating neighborhood. It is easy to see that if $V=X-\operatorname{cl}(U)$ and $A^{*}=\bigcap_{n \geq 0} f^{-n}(\operatorname{cl}(V))$, then $A^{*}$ is an attractor for $f^{-1}$ with isolating neighborhood $V$. The set $A^{*}$ is called the repeller dual to $A$. It is clear that $A^{*}$ is independent of the choice of isolating neighborhood $U$ for $A$. Obviously $f(A)=A$ and $f\left(A^{*}\right)=A^{*}$.

If we define a relation $\sim$ on $\mathbf{R}$ by $x \sim y$ if for every $\varepsilon>0$ there is an $\varepsilon>0$ there is an $\varepsilon$-chain from $x$ to $y$ and another from $y$ to $x$, then it is clear that $\sim$ is an equivalence relation.

The equivalence classes in $\mathbf{R}(f)$ for the equivalence relation $\sim$ above are called the chain transitive components of $\mathbf{R}(f)$.

(1.3) Definition. A complete Lyapounov function for $f: X \rightarrow X$ is a continuous function $g: X \rightarrow R$ satisfying:

(1) If $x \notin \mathbf{R}(f)$, then $g(f(x))<g(x)$.

(2) If $x, y \in \mathbf{R}(f)$, then $g(x)=g(y)$ iff $x \sim y$ (i.e., $x$ and $y$ are in the same chain transitive component.

(3) $g(\mathbf{R}(f))$ is a compact nowhere dense subset of $R$.

By analogy with the smooth setting, elements of $g(\mathbf{R}(f))$ are called critical values of $g$.

A theorem of C. Conley [C] asserts that a complete Lyapounov function exists for any flow or homeomorphism of a compact space. The proof in [C] is given for flows; for an exposition in the case of homeomorphisms see [F2]. 
In general the number of chain transitive components for a homeomorphism can be infinite (even uncountable). However, if we specify a fixed $\delta>0$ and work with $\delta$-chains we can decompose $R(f)$ into a finite number of pieces.

(1.4) Definition. For a fixed $\delta>0$ we say that $x, y \in \mathbf{R}(f)$ are $\delta$-equivalent if there is a $\delta$-chain from $x$ to $y$ and one from $y$ to $x$. This is an equivalence relation and the equivalence classes will be called $\delta$-transitive components of $R(f)$. We will say a compact $f$-invariant set $\Lambda \subset \mathbf{R}(f)$ is $\delta$-transitive if for every $x, y \in \Lambda, x$ is $\delta$-equivalent to $y$.

(1.5) Lemma. Given $\delta>0$ and a homeomorphism $f: X \rightarrow X$ of a compact space, then there are finitely many $\delta$-transitive components.

Proof. A $\delta$-transitive component is a union of chain transitive components. Two chain transitive components which are in different $\delta$-transitive components must be at least distance $\delta$-apart. Hence if there were infinitely many $\delta$-transitive components, there would be infinitely many subsets each at least distance $\delta$ from the others. This is impossible since $X$ is compact.

(1.6) Theorem. Given $\delta>0$ and a homeomorphism of a compact space $f: X \rightarrow$ $X$, there is a complete Lyapounov function $g: X \rightarrow R$ for $f$, and regular values for $g, c_{0}<c_{1}<c_{2}<\cdots<c_{n}$ such that if $\Lambda_{i}=\mathbf{R}(f) \cap g^{-1}\left(\left[c_{i-1}, c_{i}\right]\right)$, then $\left\{\Lambda_{i}\right\}, 1 \leq i \leq n$, are the $\delta$-transitive components of $f$.

Proof. Let $\Lambda_{1}, \ldots, \Lambda_{n}$ be the $\delta$-transitive components for $f$. We order them in such a way that if $i<j$ there is no $\delta$-chain from $\Lambda_{i}$ to $\Lambda_{j}$. This is possible since there can be no "cycle" of $\Lambda_{i}$ 's with each one having a $\delta$-chain to the next and the last having a $\delta$-chain to the first.

Let $U_{i}$ denote the set of all $z \in X$ such that there is a $\delta$-chain from $\Lambda_{i}$ to $z$. $U_{i}$ is an open set. Moreover, $f\left(\operatorname{cl}\left(U_{i}\right)\right) \subset U_{i}$, because if $z \in \operatorname{cl}\left(U_{i}\right)$, there is $z_{0} \in U_{i}$ such that $d\left(f(z), f\left(z_{0}\right)\right)<\delta$ and consequently a $\delta$-chain from $x$ to $z_{0}$ gives a $\delta$-chain $x=x_{1}, x_{2}, \ldots, x_{k}, z_{0}, f(z)$ from $x$ to $f(z)$.

Thus if $A_{i}=\bigcap_{n \geq 0} f^{n}\left(\operatorname{cl} U_{i}\right)$ and $A_{i}^{*}=\bigcap_{n \geq 0} f^{-n}\left(X-U_{i}\right)$, then $A_{i}, A_{i}^{*}$ are an attractor repeller pair and $\Lambda_{i} \subset A_{i}$. A result of Conley (see Lemma (1.7) of [F2] for a proof) asserts there is a continuous function $g_{i}: X \rightarrow[0,1]$ such that $A_{i}=g_{i}^{-1}(0), A_{i}^{*}=g_{i}^{-1}(1)$ and $g_{i}(f(x))<g_{i}(x)$ for all $x \in X-\left(A_{i} \cup A_{i}^{*}\right)$. If $i<j$, then $\Lambda_{j} \subset A_{i}^{*}$ so $g_{i}\left(\Lambda_{j}\right)=\{1\}$.

Let $h(x)=\sum_{i=1}^{n} 2^{i} g_{i}(x)$ and note that $h(f(x)) \leq h(x)$ for all $x \in X$. For $x \in \mathbf{R}(f)=\bigcup \Lambda_{i}, h(x)$ is an even integer between 0 and $2^{n+1}$. Also note if $x, y \in \mathbf{R}(f)$, then $h(x)=h(y)$ if and only if $g_{i}(x)=g_{i}(y)$ for all $i$. Hence if $x \in \Lambda_{i}, y \in \Lambda_{j}, i<j$, then $h(x) \neq h(y)$ since $g_{i}(x) \neq g_{i}(y)$. Now if $g_{0}: X \rightarrow[0,1]$ is a complete Lyapounov function, then $g(x)=g_{0}(x)+h(x)$ is the desired function. 


\section{The $\delta$-TRANSITIVE CASE}

We begin with a sequence of results leading to our main theorem. Assume throughout that $f: T^{2} \rightarrow T^{2}$ is a homeomorphism homotopic to the identity and $F: R^{2} \rightarrow R^{2}$ is a lift, i.e., if $\pi: R^{2} \rightarrow T^{2}$ is the covering projection then $\pi \circ F=f \circ \pi$.

(2.1) Lemma. If $F$ has no fixed points, then there is an $\varepsilon>0$ such that no periodic $\varepsilon$-chain for $F$ exists.

Proof. This result and its proof are quite similar to (2.1) of [F1] and (2.2) of [F2]. Let

$$
\delta=\min _{x \in R^{2}}|F(x)-x|
$$

Note this minimum is assumed since it suffices to consider only $x$ in a compact fundamental domain for $\pi$. Hence $\delta>0$.

A result of Oxtoby [Ox] says that there is a $\gamma>0$ such that for any finite set of pairs $\left\{\left(x_{i}, y_{i}\right)\right\}$ of elements in $R^{2}$ with $\left\|x_{i}-y_{i}\right\|<\gamma$ there is a pairwise disjoint set of piecewise linear arcs $\alpha_{i}$ from $x_{i}$ to $y_{i}$ with the diameter of each $<\delta$.

Let $\varepsilon=\gamma$; we will show there is no periodic $\varepsilon$-chain for $F$. Suppose to the contrary that $z_{1}=z, z_{2}, z_{3}, \ldots, z_{n}=z$ is a periodic $\varepsilon$-chain. Letting $y_{i}=z_{i}, x_{i}=F\left(z_{i-1}\right)$, we see that there are pairwise disjoint arcs $\alpha_{i}$ from $F\left(z_{i-1}\right)$ to $z_{i}$, with diameter $<\delta$. By isotoping in a neighborhood of these arcs we can produce a perturbation $G$ of $F$ satisfying

(1) $\|F(x)-G(x)\|<\delta$ for all $x \in R^{2}$, and

(2) $G\left(z_{i-1}\right)=z_{i}$.

Now $G$ has a periodic point, namely $z$. Hence by results of [Br or Fa] $G$ has a fixed point $p$. Thus $\|F(p)-p\| \leq\|F(p)-G(p)\|+\|G(p)-p\|<\delta$ which is a contradiction.

(2.2) Lemma. Suppose $\Lambda$ is a $\delta$-transitive compact invariant subset of $\mathbf{R}(f)$ for a homeomorphism $f: T^{2} \rightarrow T^{2}$ and $F$ is a lift of $f$. There is a constant $K>0$, such that for any $x_{0}, y_{0} \in \Lambda, x \in \pi^{-1}\left(x_{0}\right)$ there is a $\delta$-chain for $F$ from $x$ to a point $y \in \pi^{-1}\left(y_{0}\right)$ with $\|y-x\|<K$.

Proof. Fix $\omega \in \pi^{-1}(\Lambda)$ and let $Q_{n}$ denote the set of $z \in \Lambda$ such that there is a $\delta$-chain for $f$ from $\pi(\omega)$ to $z$ of length less than $n . Q_{n}$ is open by definition and $\Lambda=\bigcup_{n \geq 1} Q_{n}$ so compactness of $\Lambda$ implies $Q_{N}=\Lambda$ for some $N>0$. Hence given $y_{0} \in \Lambda$ there is a $\delta$-chain from $\pi(\omega)$ to $y_{0}$ of length less than $N$. Lifting this to $R^{2}$, starting at $w$, we obtain a $\delta$-chain from $w$ to some $y^{\prime} \in \pi^{-1}\left(y_{0}\right)$. If $P=\sup \|F(\nu)-\nu\|$, then since this $\delta$-chain from $w$ to $y^{\prime}$ has length less than $N$, it follows that $\left\|y^{\prime}-w\right\|<C_{1}=N(P+\delta)$.

A similar argument shows that given $x_{0} \in \Lambda$ there is an $x^{\prime} \in \pi^{-1}\left(x_{0}\right)$ with a $\delta$-chain from $x^{\prime}$ to $w$ and $\left\|x^{\prime}-w\right\|<C_{2}$ for some constant $C_{2}$ independent of 
$x_{0}$. Piecing these together we obtain a $\delta$-chain from $x^{\prime}$ to $y^{\prime}$ with $\left\|y^{\prime}-x^{\prime}\right\|<$ $K=C_{1}+C_{2}$. Now given any $x \in \pi^{-1}\left(x_{0}\right)$ translate this $\delta$-chain by the integer vector $x-x^{\prime}$ to obtain a $\delta$-chain from $x$ to $y$, where $y=y^{\prime}+\left(x-x^{\prime}\right)$ satisfies $\pi(y)=y_{0}$ and $\|y-x\|=\left\|y^{\prime}-x^{\prime}\right\|<K$.

(2.3) Definition. If $\Lambda \subset T^{2}$ is a compact invariant set for $f: T^{2} \rightarrow T^{2}$, and $F$ is a lift of $f$, we denote by $\rho(f, \Lambda)$, the accumulation points of the set

$$
\left\{\frac{F^{n}(x)-x}{n} \mid \pi(x) \in \Lambda \text { and } n>0\right\} .
$$

(2.4) Proposition. Suppose $\Lambda \subset T^{2}$ is a compact invariant subset of $\mathbf{R}(f)$ for $f: T^{2} \rightarrow T^{2}$ and for some $\delta>0, \Lambda$ is $\delta$-transitive. If 0 is in the interior of the convex hull of $\rho(F, \Lambda)$, then there is a periodic $\delta$-chain for $F$.

Proof. The hypothesis guarantees that there are vectors $\nu_{1}, \nu_{2}, \nu_{3}, \nu_{4} \in \rho(F, \Lambda)$ such that 0 is in the interior of their convex hull (see Steinitz's theorem in [HDK]). Choose neighborhoods $U_{i}$ of $\nu_{i}$ in $R^{2}$ so small that whenever $\nu_{i}^{\prime} \in$ $U_{i}, 0$ is also in the interior of the convex hull of $\nu_{1}^{\prime}, \nu_{2}^{\prime}, \nu_{3}^{\prime}$ and $\nu_{4}^{\prime}$. Fix $z_{0} \in \Lambda$ and $z \in \pi^{-1}\left(z_{0}\right)$. Now by (2.2) and the fact that $\nu_{1} \in \rho(F, \Lambda)$ we can find $x_{i} \in R^{2}$ and $n_{i}>i$ such that

(1) $\lim _{i \rightarrow \infty} \frac{F^{n_{i}}\left(x_{i}\right)-x_{i}}{n_{i}}=\nu_{1}$.

(2) There is a $\delta$-chain from $z$ to $x_{i}$ and $\left\|x_{i}-z\right\|<K$.

(3) There is a $\delta$-chain from $F^{n_{i}}\left(x_{i}\right)$ to $z_{i}^{\prime} \in \pi^{-1}\left(z_{0}\right)$ and $\left\|F^{n_{i}}\left(x_{i}\right)-z_{i}^{\prime}\right\|<$ $K$.

Notice that piecing together the $\delta$-chain from $z$ to $x_{i}$, the orbit segment from $x_{i}$ to $F^{n_{i}}\left(x_{i}\right)$ and the $\delta$-chain from $F^{n_{i}}\left(x_{i}\right)$ to $z_{i}^{\prime}$ we obtain a $\delta$-chain from $z$ to $z_{i}^{\prime}$. Also (1), (2), and (3) imply

$$
\lim _{n \rightarrow \infty} \frac{z_{i}^{\prime}-z}{n_{i}}=\nu_{1}
$$

Choose $i$ sufficiently large that

$$
\frac{z_{i}^{\prime}-z}{n_{i}} \in U_{1}
$$

and set $w_{1}=z_{i}^{\prime}-z, m_{1}=n_{i}$ so that there is a $\delta$-chain from $z$ to $z+w_{1}$ and $w_{1} / m_{1} \in U_{1}$. Note that $\pi\left(z_{i}^{\prime}\right)=\pi(z)=z_{0}$ implies $w_{1}$ is an integer vector.

Now in a similar fashion construct $w_{2}, m_{2}, w_{3}, m_{3}$, and $w_{4}, m_{4}$, with the analogous properties.

Since 0 is in the convex hull of $w_{1} / m_{1}, w_{2} / m_{2}, w_{3} / m_{3}, w_{4} / m_{4}$ and the vectors $w_{1}, w_{2}, w_{3}, w_{4}$ are integers, it is possible to solve

$$
A w_{1}+B w_{2}+C w_{3}+D w_{4}=0
$$


for positive integers $A, B, C, D$. Any translate of a $\delta$-chain by an integer vector is another $\delta$-chain. Hence piecing together $A$ translates of the $\delta$-chain from $z$ to $z+w$, with $B$ translates of the $\delta$-chain from $z$ to $z+w_{2}, C$ translates of the $\delta$-chain from $z$ to $z+w_{3}$, etc., we obtain a $\delta$-chain from $z$ to $z+A w_{1}+B w_{2}+C w_{3}+D w_{4}=z$ as desired.

\section{THE General CASE}

As before we assume $f: T^{2} \rightarrow T^{2}$ is a homeomorphism and $F: R^{2} \rightarrow R^{2}$ is a lift.

(3.1) Proposition. Suppose $\nu_{1}, \nu_{2}, \nu_{3}$ and $\nu_{4}$ are extreme points of the convex set $\rho(F)$ and 0 is in the interior of their convex hull. Then $F$ possesses a fixed point.

Proof. In [MZ] it is shown that since $\nu_{i}$ is an extreme point of $\rho(F)$ there is an ergodic Borel measure realizing $\nu_{i}$ and hence a nonwandering point $x_{i} \in T^{2}$ such that if $x \in \pi^{-1}\left(x_{i}\right)$

$$
\lim _{n \rightarrow \infty} \frac{F^{n}(x)-x}{n}=\nu_{i}
$$

We will need only the fact that such an $x_{i}$ exists with $x_{i} \in \mathbf{R}(f)$.

To show that $F$ has a fixed point it suffices by $(2.1)$ to show that for every $\delta>0$ there is a periodic $\delta$-chain for $F$. Given $\delta>0$, let $\mathbf{R}(f)=\Lambda_{1} \cup$ $\Lambda_{2} \cup \cdots \cup \Lambda_{m}$ be a decomposition of the chain recurrent set into $\delta$-transitive pieces as given in (1.6) and let $g: T^{2} \rightarrow R$ be a complete Lyapounov function compatible with this decomposition. We will show that there exists a piece $\Lambda_{j}$ of this decomposition and points $y_{i} \in \Lambda_{j}, i=1,2,3,4$, such that whenever $y \in \pi^{-1}\left(y_{i}\right)$,

$$
\nu_{i}=\lim _{n \rightarrow \infty} \frac{F^{n}(y)-y}{n} .
$$

It then follows by (2.4) that $F$ has a $\delta$-chain. Since this holds for all $\delta>0$ we conclude by (2.1) that $F$ has a fixed point.

Choose a smooth approximation $g_{0}: T^{2} \rightarrow R$ to $g$ and regular values $c_{1}, c_{2}, \ldots, c_{m}$ such that the manifolds with boundary $M_{i}=g_{0}^{-1}\left(\left(-\infty, c_{i}\right]\right)$ satisfy

(1) $f\left(M_{i}\right) \subset$ int $M_{i}$, and

(2) $\Lambda_{i} \subset M_{i}-M_{i-1}$.

Let $N_{i}$ be the manifold $\operatorname{cl}\left(M_{i}-M_{i-1}\right)$, so $T^{2}=\bigcup N_{i}$ and $N_{i} \cap N_{k}$ consists of a finite set of circles if $k=i \pm 1$ and otherwise is empty if $i \neq k$.

These circles are the components of $g_{0}^{-1}\left(\left\{c_{1}, c_{2}, \ldots, c_{m}\right\}\right)$. We first observe that none of these circles is essential in $T^{2}$. If there were such a circle, say 
$\gamma$, then it would be in the boundary of $M_{j}$ for some $j$ and $M_{j}$ would have to have another boundary component which is isotopic to $\gamma$. (There might also be some inessential circles in the boundary of $M_{j}$.) It follows that $M_{j}$ is an essential annulus (perhaps with some disks removed) in $T^{2}$. Let $\tilde{M}_{j}$ be a component of $\pi^{-1}\left(M_{j}\right)$ and choose a lift $F_{0}$ of $f$ so that $F_{0}\left(\tilde{M}_{j}\right) \subset \tilde{M}_{j}$. Now $\tilde{M}_{j}$ is an infinite strip (perhaps with holes) which has a rational slope. It follows since $F_{0}\left(\tilde{M}_{j}\right) \subset \tilde{M}_{j}$ that for any $x \in R^{2}$, if $\lim _{n \rightarrow \infty} \frac{F^{n}(x)-x}{n}$ exists, then it must lie on a line with this slope, since $F_{0}^{n}(x)$ is constrained between parallel translates of $\tilde{M}_{j}$. From this and the fact that $F(x)=F_{0}(x)+w$ for some integer vector $w$, it follows that the convex hull of the vectors $\nu_{i}$ given in our hypothesis is a line segment. This contradicts the assumption that 0 is in the interior of the convex hull; so none of the boundary components of the $N_{i}$ can be essential in $T^{2}$.

Since each of these boundary circles is inessential, each of them bounds a unique smooth disk in $T^{2}$. The complement of the union of these disks consists of the interior of a single one of the $N_{i}$ 's, say $N_{j}$. The complement of $\operatorname{int}\left(N_{j}\right)$ in $T^{2}$ consists of a finite set of disks, say $D_{1}, D_{2}, \ldots, D_{r}$. Number these disks so that

$$
D_{i} \subset M_{j} \quad \text { for } 1 \leq i \leq s
$$

and

$$
D_{i} \subset \operatorname{cl}\left(T^{2}-M_{j}\right) \quad \text { for } s<i \leq r
$$

Then

$$
f\left(D_{i}\right) \subset \bigcup_{k=1}^{s} D_{k} \quad \text { for } 1 \leq i \leq s
$$

and

$$
f^{-1}\left(D_{i}\right) \subset \bigcup_{k=s+1}^{r} D_{k} \quad \text { if } s<i \leq r .
$$

Consider now a point $x \in \pi^{-1}\left(x_{1}\right)$ such that

$$
\nu_{1}=\lim _{n \rightarrow \infty} \frac{F^{n}(x)-x}{n} .
$$

We will show that if $x_{1}$ is not in $\Lambda_{j}$, there is another point $y_{1} \in \Lambda_{j}$ so that whenever $y \in \pi^{-1}\left(y_{1}\right)$,

$$
\nu_{1}=\lim _{n \rightarrow \infty} \frac{F^{n}(y)-y}{n} .
$$

Since the same is true for $\nu_{2}, \nu_{3}$, and $\nu_{4}$, we will have completed the proof by the remarks above.

Suppose now that $x_{1} \in D_{p}$ for $1 \leq p \leq s$. There exists $q>0$ such that $f^{q}\left(D_{p}\right) \subset D_{p}$ (recall that $x_{1}$ is recurrent). Hence if $D \subset R^{2}$ is the lift of 
$D_{p}$ containing $x$, then $F^{q}(D) \subset D+w$ for some integer vector $w$. If we set $G(z)=F^{q}(z)-w$, then $G(D) \subset D$ so there is a fixed point $z_{0}$ for $G$. Clearly

$$
\nu_{1}=\lim _{n \rightarrow \infty} \frac{F^{n}(x)-x}{n}=\lim _{n \rightarrow \infty} \frac{F^{n}\left(z_{0}\right)-z_{0}}{n}=\frac{w}{q} .
$$

If $x_{1} \in D_{p}$ and $s<p$, then a similar argument applied to $f^{-1}$ leads to a fixed point $z_{0}$ of $G$ with the same properties.

We want to find a fixed point for $G$ which is in $\pi^{-1}\left(N_{j}\right)$. To do this we consider fixed points of $f^{q}$ on $T^{2}$. We will use the fact that $f^{q}$ is homotopic to a map with no fixed points so the index sum of the set of fixed points in any Nielsen class for $f^{q}$ is zero (see [B, Theorem 3, p. 94]). Recall that two fixed points $p_{1}$ and $p_{2}$ are in the same Nielsen class for $f^{q}$ provided any lift of $f^{q}$ to $R^{2}$ which pointwise fixes $\pi^{-1}\left(p_{1}\right)$ also pointwise fixes $\pi^{-1}\left(p_{2}\right)$.

We will consider points in the Nielsen class of the point $\pi\left(z_{0}\right)$ where $z_{0}$ is the fixed point of $G$ mentioned above. Any such points which are not in $N_{j}$ will lie in a $D_{i}$ with a lift $\tilde{D}_{i}$ for which $G\left(\tilde{D}_{i}\right) \subset \tilde{D}_{i}$ or with $G^{-1}\left(\tilde{D}_{i}\right) \subset \tilde{D}_{i}$. Hence the contribution to the index of the points in $D_{i}$ will be +1 . Thus the index of the set of fixed points in the Nielsen class of $\pi\left(z_{0}\right)$ which are not in $N_{j}$ is positive (the disk $D_{p}$ contributes at least one +1 ). It follows there must be a fixed point $y_{1} \in N_{j}$ of $f^{q}$ in the Nielsen class of $\pi\left(z_{0}\right)$. Since $y_{1}$ is in the Nielsen class of $\pi\left(z_{0}\right)$, if $y \in \pi^{-1}\left(y_{1}\right)$, then $G(y)=y$. Hence

$$
\nu_{1}=\lim _{n \rightarrow \infty} \frac{F^{n}(y)-y}{n} \text {. }
$$

Also $y_{1}$ is a periodic point of $f$ in $N_{j}$ so $y_{1} \in \Lambda_{j}$. The same argument implies the existence of $y_{2}, y_{3}, y_{4} \in \Lambda_{j}$, so this completes the proof.

(3.2) Theorem. Suppose $f: T^{2} \rightarrow T^{2}$ is a homeomorphism homotopic to the identity and $F: R^{2} \rightarrow R^{2}$ is a lift. If $\nu$ is a vector with rational coordinates in the interior of $\rho(F)$, then there is a point $p \in R^{2}$ such that $\pi(p) \in T^{2}$ is a periodic point for $f$ and

$$
\nu=\lim _{n \rightarrow \infty} \frac{F^{n}(p)-p}{n} .
$$

Proof. Suppose $\nu=(r / q, s / q)$ with the greatest common divisor of $r, s$, and $q$ equal to 1 . If $G(x)=F^{q}(x)-(r, s)$, then a fixed point $p$ of $G$ will satisfy $F^{q}(p)=p+(r, s)$ and hence be the desired point.

It is easy to check (see [MZ]) that $\rho(G)=q \rho(F)-(r, s)$. Thus since $(r / q, s / q)$ is in the interior of $\rho(F)$, it follows that 0 is in the interior of $\rho(G)$. Since $\rho(G)$ is closed and convex there exist extreme points $\nu_{1}, \nu_{2}, \nu_{3}, \nu_{4} \in \rho(G)$ such that 0 is in their convex hull (see Steinitz's theorem in [HDK]). It now follows from (3.1) that $G$ possesses a fixed point $p$. 


\section{REFERENCES}

[B] R. F. Brown, The Lefschetz fixed point theorem, Scott Foresman and Co., Glenview, Ill., 1978.

[Br] M. Brown, A new proof of Brouwer's lemma on translation arcs, Houston J. Math. 10 (1984), $35-41$.

[C] C. Conley, Isolated invariant sets and the Morse index, CBMS Regional Conf. Ser. in Math., no. 38, Amer. Math. Soc., Providence, R. I., 1978.

[Fa] A. Fathi, An orbit closing proof of Brouwer's lemma on translation arcs, Enseign. Math. 33 (1987), 315-322.

[F] J. Franks, Recurrence and fixed points of surface homeomorphisms, Ergodic Theory Dynamical Systems $8^{*}$ (1988), 99-107.

[F2] __ A variation on the Poincaré-Birkhoff theorem, Hamiltonian Dynamics, Contemp. Math., Amer. Math. Soc., Providence, R. I. (to appear).

[HDK] H. Hadwiger, H. Debrunner, and V. Klee, Combinatorial geometry in the plane, Holt Rinehart and Winston, New York, 1964.

[ML] R. MacKay and J. Llibre, Rotation vectors and entropy for homeomorphisms homotopic to the identity, preprint.

[MZ] M. Misiurewicz and K. Ziemian, Rotation sets of toral maps (to appear).

[Ox] J. Oxtoby, Diameters of arcs and the gerrymandering problem, Amer. Math. Monthly 84 (1977), 155-162.

Department of Mathematics, Northwestern University, Evanston, Illinois 60201 